\title{
Traitement orthodontique dans les fentes labio-palatines au Mexique
}

\author{
Eric Beljour ${ }^{1}{ }^{*}$, Mónica Domínguez ${ }^{2}$, María del Carmen Rosas Ramírez², \\ Manuel Yudovich Burak ${ }^{2}$ \\ ${ }^{1}$ Département d'Orthodontie, UNAM, et Division de Stomatologie-Orthodontie, Hôpital général Dr Manuel Gea González, \\ Mexico, Mexique \\ 2 Division de Stomatologie-Orthodontie, Hôpital général Dr Manuel Gea González, Mexico, Mexique
}

(Reçu le 3 septembre 2012, accepté le 2 octobre 2012)

Mots clés :

fente labio-palatine /

orthopédie pré-

chirurgicale /

traitement

orthodontique /

orthopédie maxillaire
Résumé - Les patients ayant une fente labio-palatine (FLP) présentent diverses anomalies maxillo-faciales, associées à un faible potentiel de croissance et de développement des structures. En fonction de leurs manifestations phénotypiques, les FLP sont classées en formes modérées ou sévères. Pendant toutes les étapes de la croissance, les patients doivent être intégrés dans un programme individualisé de contrôle et de stimulation. Par ailleurs, les interventions chirurgicales peuvent comporter des séquelles qui majorent parfois certaines anomalies. Le traitement orthodontique est donc nécessaire et même capital pour obtenir les résultats désirés et assurer un état final harmonieux.

Cet article présente un ensemble de mesures (interventions d'orthodontie et d'orthopédie maxillaire) dont l'application permet d'aboutir aux objectifs correspondant aux différentes étapes du développement du sujet et qui s'intègre dans le traitement golbal.

Key words: cleft lip and palate / presurgical orthopedic management / orthodontic treatment / maxillofacial orthopedic
L'orthodontiste joue un rôle fondamental dans le traitement intégré des patients ayant des malformations craniomaxillo-faciales. Il contribue très tôt à la réhabilitation des patients ayant une fente labio-palatine (FLP) ou une autre anomalie dento-faciale. Il stimule la croissance et le développement des structures formant cette région, en utilisant des appareils orthopédiques fonctionnels. Par la suite, avec le traitement orthodontique, il complète le traitement des autres spécialistes du groupe multidisciplinaire participant à la prise en charge de ces patients. Pour les étapes tardives (patients n'ayant pas bénéficié d'une prise en charge précoce), il contribue à la réhabilitation et cherche à obtenir un état stable à long terme du patient et l'amène à un état de santé physique et mental optimal [1-6].

\section{Objectifs}

On doit s'efforcer de soigner et de traiter tous les patients ayant une FLP pour empêcher le développement de troubles de l'occlusion dentaire, afin de prévenir ou de diminuer les manifestations et les séquelles anatomiques et fonctionnelles secondaires.

\footnotetext{
*Correspondance : orthodontie.haiti@yahoo.fr
} 


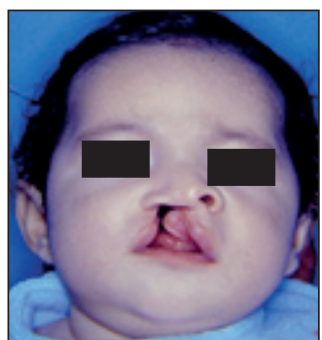

a

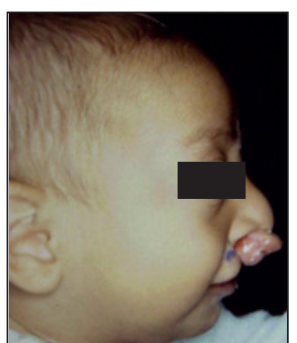

b

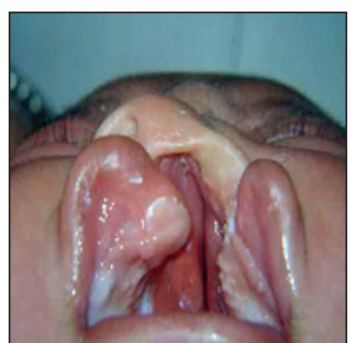

C

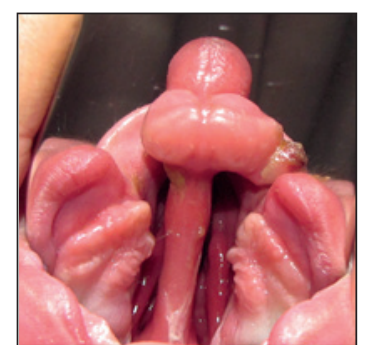

d

Fig. 1. A : vue de face, $B:$ vue de profil, $C:$ vue inférieure, $D:$ vue intra-buccale. Fig. 1. A: frontal view, $B$ : lateral view, C: basal view, $D$ : intraoral view.

L'orthopédie fonctionnelle permet de réorienter la croissance de la région dento-faciale et d'obtenir une relation harmonieuse entre les os de la face donnant ainsi au sujet une occlusion fonctionnelle et esthétique stable. Avec un bon contrôle de la croissance, on pérennise le résultat des interventions chirurgicales et on obtient un équilibre optimum des maxillaires. La comparaison des l'état initial et de l'état final permet d'évaluer l'efficacité du traitement [1-6].

\section{Étape initiale : étude et traitement}

\section{Orthopédie pré-chirurgicale}

Pendant les premiers mois, avant l'intervention de chirurgie réparatrice, l'orthopédie permet de stimuler et de mobiliser les segments maxillaires. Elle a pour objectif de repositionner, d'aligner et de réduire les segments maxillaires en les ramenant à une distance minimale afin de pouvoir effectuer une fermeture chirurgicale dans les meilleures conditions. Cette façon de procéder offre plusieurs avantages : elle permet de réaliser une fermeture de la lèvre sans tension, de restaurer le contour normal de l'arcade maxillaire, de faciliter l'alimentation er de repositionner les cartilages des ailes du nez et de la cloison nasale [7-11]. Cette technique met à profit la malléabilité des cartilages et des segments osseux pendant les premières semaines de la vie.

L'établissement d'un diagnostic initial très précoce permet d'identifier le type de FLP et de choisir les mesures thérapeutiques spécifiques pour le cas. La première étape est celle de l'orthopédie pré-chirurgicale qui s'applique de la naissance au 3ème mois. Dès la première visite, on procède á la prise des photographies extra et intra-buccales et à la prise d'empreinte pour la confection de modèles d'étude du procès alvéolaire supérieur. Des photographies extra-buccales sont prises systématiquement de face, de profil des deux côtés et en vue inférieure; les photographies intra-buccales sont prises avec un miroir ce qui donne une bonne vue d'ensemble de la FLP (Fig. 1). Les modèles permettent d'affiner le diagnostic et de fabriquer l'appareil indiqué. Pour les empreintes, on utilise un élastomère, le polyvinyle de siloxane, car c'est un produit facile à contrôler ; le patient doit être placé dans une position inclinée, la tête en bas, pour éviter d'éventuelles complications par obstruction des voies respiratoires.

Lors de l'auscultation, on doit considérer trois régions anatomiques spécifiques : le nez, le palais primaire et le palais secondaire. Le cartilage alaire constitue le cartilage le plus affecté ; il est déplacé dans les trois plans de l'espace, de façon proportionnelle à la taille de la fente. On distingue trois degrés selon l'importance de la fente [4]. Dans le degré léger, on observe un déplacement horizontal vers le bas et en dehors de la pointe et de l'aile du nez du côté de la fente. Dans le degré modéré, un déplacement dans le plan horizontal et le plan vertical vers le bas, en dessous du plancher nasal, du côté de la fente. Dans le degré sévère, un déplacement dans les trois plans (horizontal, vertical et frontal) de la pointe et de l'aile du nez du côté de la fente. On y ajoute un composé septal lequel se trouve dévié à un degré allant de léger à modéré. Pour le palais primaire, on mesure la distance entre les bords de la FLP ; ceux-ci peuvent être alignés ou effondrés. Pour une FLP bilatérale, la distance entre le bord du maxillaire et le bord du prémaxillaire de chaque côté. Pour le palais secondaire, la distance entre les segments palatins à hauteur de la limite palais dur-palais mou.

Pour réaliser le traitement d'orthopédie pré-chirurgical, on dispose de plusieurs appareils : plaques passives, plaques de pression sélective, appareil de Grayson, appareils pour étendre et élargir les orifices narinaires... Chaque appareil possède des indications spécifiques mais le choix dépend aussi des habitudes du praticien [7-11] (Fig. 2). L'appareil choisi doit être utilisé pendant trois mois et rester en bouche 10 heures par jour. Toutes les semaines, l'enfant est revu en consultation afin de réajuster l'appareil ou de le changer si nécessaire.

Avant la chéiloplastie, on répète à nouveau toute la procédure initiale : prise de photographies, mesure de la taille de la FLP pour vérifier l'efficacité du traitement et réévaluer le patient avant l'intervention chirurgicale. Après la chéiloplastie, les consultations restent fréquentes (une fois pas semaine) pour surveiller si la pression secondaire au processus 


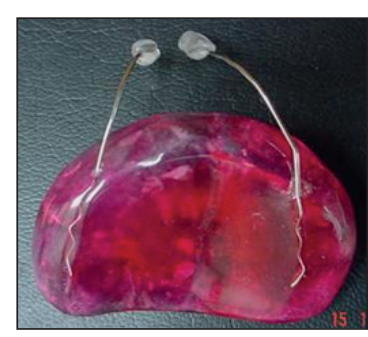

a

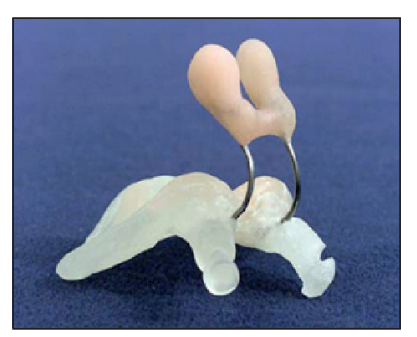

b

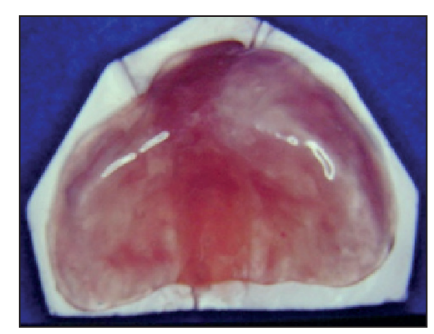

C

Fig. 2. A : plaque de pression sélective avec extensions nasales, B : appareil de Grayson, $C$ : plaque passive. Fig. 2. A: selective pressure device with nasal stents, B: Grayson's molding plate, C: passive plate.

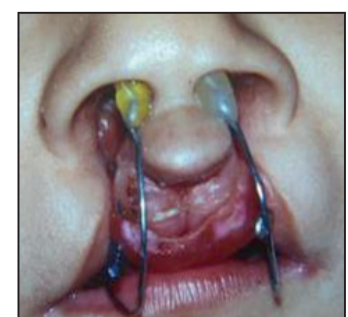

a

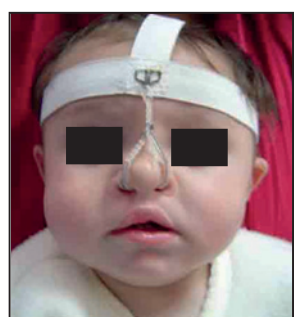

b

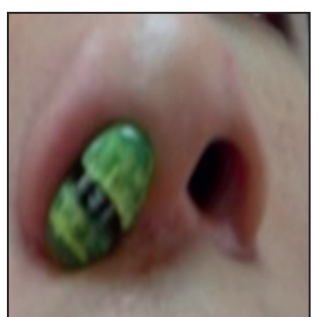

C

Fig. 3. A : plaque de contention avec extensions nasales, B : conformateur nasal bilatéral avec appui céphalique, $\mathrm{C}:$ conformateur avec vis d'expansion.

Fig. 3. A: retention plate with nasal stents, B: bilateral nasal splint with cranial support, C: splint with expansion screw.

de cicatrisation n'affecte pas la croissance et le développement des structures.

L'étape suivante s'étend normalement du troisième au douzième mois. 0 n prend à nouveau des photos intra et extrabuccales et des empreintes pour réaliser des modèles d'étude. Les modèles sont utilisés pour la fabrication des appareils d'orthodontie et des mainteneurs d'espace. L'objectif de cette étape est de contenir les segments palatins, réduire la taille de la fente palatine et de tonifier les muscles pour rapprocher les segments palatins $[12,13]$.

L'auscultation nasale se fait de la même façon que lors de la première consultation. Pour allonger ou étendre le pilier et les orifices nasaux, et redonner au contour nasal sa forme, on utilise la plaque de Grayson, un appareil fait avec du fil orthodontique TMA 0.32 ou une plaque passive en résine acrylique avec deux extensions nasales en fil d'acier inoxydable, portant des conformateurs avec une vis. On utilise cette plaque quand il y a un effondrement important du nez. On active le vis une fois par semaine. L'appareil est utilisé tous les jours jusqu'à la fermeture chirurgicale du palais et on cesse d'activer la vis d'expansion nasale lorsqu'on a obtenu la symétrie des orifices narinaires. Il est recommandé aux parents de laver l'appareil tous les jours avec de l'eau et du savon (Fig. 3).

Le patient est vu en contrôle tous les mois et, à chaque consultation, on évalue la forme, la position et la direction de l'aile narinaire du côté affecté, le pilier nasal et la fente.
En fonction de l'évolution et de l'avis de l'équipe interdisciplinaire, on programme la $2^{\mathrm{e}}$ intervention chirurgicale, la plastie palatine, qui comporte ou non un temps pharyngé.

\section{Période post-palatoplastie}

Après la fermeture du palais, on répète les mêmes procédures, en particulier la prise des photographies intra-buccales pour repérer une éventuelle anomalie ou fistule; des photographies extra-buccales pour évaluer la taille du pilier nasal et la symétrie des orifices narinaires. L'objectif est de maintenir la stabilité des segments maxillaires et de la forme du nez. Pour réaliser ces objectifs, on utilise une plaque acrylique de contention avec des extensions nasales. Il est recommandé de l'utiliser tous les jours et les contrôles se font tous les mois.

\section{Orthopédie maxillaire}

En général, cette étape s'étend de 2 ans à 5 ans. De nouveau, on effectue un suivi photographique et des radiographies panoramiques et du crâne de profil. On réalise des modèles d'étude (Fig. 4) pour suivre l'évolution de l'occlusion : on évalue la forme de l'arcade dentaire, la présence d'occlusion croisée antérieure et/ ou postérieure, les distances intermolaire et inter-canine, les classes molaire et canine (I, II et III), l'over-jet, l'over-bite et la profondeur du palais. 

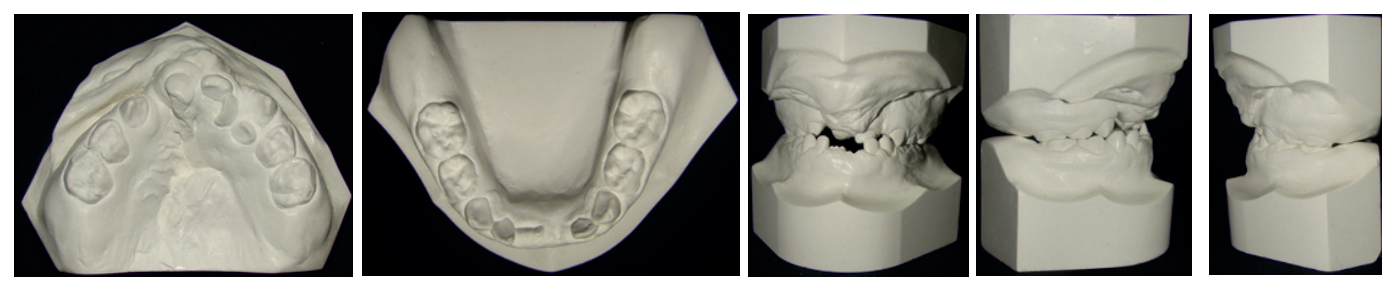

Fig. 4. Modèle d'étude.

Fig. 4. Study model.

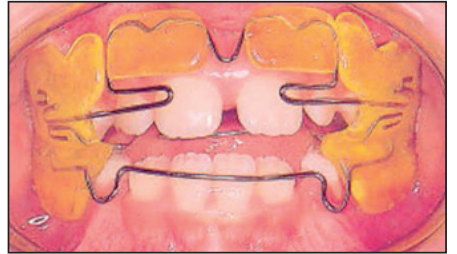

a

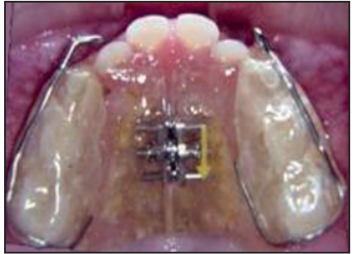

b

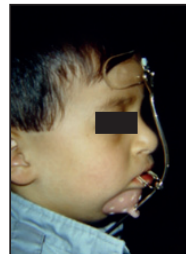

C

Fig. 5. A : régulateur de fonction de Franckel, B : plaque d'expansion avec recouvrement occlusal et crochets pour le masque facial, C: masque facial.

Fig. 5. A: Frankel functional regulator, B: expansion plate with occlusal covering and hooks for face mask, C: face mask.

Sur la radiographie panoramique, on recherche la présence ou non de dents surnuméraires et/ou ectopiques, d'agénésie, on évalue la forme du corps et des ramus mandibulaires, la symétrie et le parallélisme des condyles. Sur la téléradiographie de profil, les relations antéro-postérieure et verticale des maxillaires entre eux et avec la base du crâne.

L'objectif général est de corriger les décalages antéropostérieur, frontal et vertical du maxillaire et de la mandibule. Pour ce, on utilise un appareil intra-oral avec une vis d'expansion et des crochets en fil d'acier inoxydable, placés dans la région des canines et qui ont des supports dans la région molaire et au palais, pour adapter le masque facial. On peut aussi utiliser un appareil de stimulation musculaire (myo-fonctionnel) $[12,13]$ pour corriger le décalage inter-maxillaire, rééduquer la musculature péribuccale dans le cas où il existe des anomalies des praxies fonctionnelles. Il est recommandé d'utiliser le masque facial 12 à 14 heures par jour, et l'activation de la vis se fait en fonction du cas et de l'appareil (Fig. 5). On effectue une consultation mensuelle pour évaluer l'évolution et ajuster ou modifier l'appareil si nécessaire.

\section{Greffe osseuse}

Cette intervention chirurgicale est effectuée entre 6 et 11 ans. En plus des éléments habituels, on doit disposer d'une radiographie occlusale et, dans certains cas, d'une tomographie. Sur la téléradiographie de profil, on réalise le tracé de l'analyse céphalométrique pour compléter le diagnostic. Il existe plusieurs types d'analyse céphalométrique (Ricketts, Jarabak, Tweed, Steiner...). Elle permet de diagnostiquer la position et la relation des maxillaires avec la base du crâne
(14-17). Les normes les plus utilisées sont celles de Steiner, en particulier l'angle SNA (selle turcique-nasion-point $A$ ), l'angle SNB (selle turcique-nasion-point B) et l'angle ANB qui donne la relation entre le maxillaire et la mandibule, donc le type de la classe squelettique (Fig. 6).

Sur la radiographie panoramique (Fig. 7), on examine la présence des dents et leur taille pour décider de réaliser, si nécessaire, certaines extractions pour créer de l'espace. La radiographie occlusale donne la position et la forme des canines supérieures, et la taille de la fente après la fermeture du palais.

Avec le traitement orthodontique, on cherche à aligner les dents permanentes présentes en bouche mais aussi à corriger le décalage sagittal, frontal et vertical maxillo-mandibulaire avant de réaliser la greffe osseuse alvéolaire secondaire. Pour ce traitement, on utilise des appareils intra-oraux ayant des supports dentaires et muqueux avec des vis d'expansion et des crochets pour utiliser éventuellement le masque facial. On peut aussi utiliser des appareils type Franckel ou Bimler et/ou des appareils orthodontiques classiques. Le traitement dure jusqu'à obtention d'une relation et d'une position adéquates du maxillaire et de la mandibule. Les consultations sont mensuelles et, à la fin de cette étape, on évalue une nouvelle fois les relations dentaires et squelettiques [18-20].

\section{Orthodontie conventionnelle et orthodontie pré-chirurgi- cale tardive}

Cette phase s'étend de 12 à 16 ans. Il est nécessaire comme pour les étapes précédentes, de faire une évaluation complète avec tous les éléments de diagnostic nécessaires comme les 

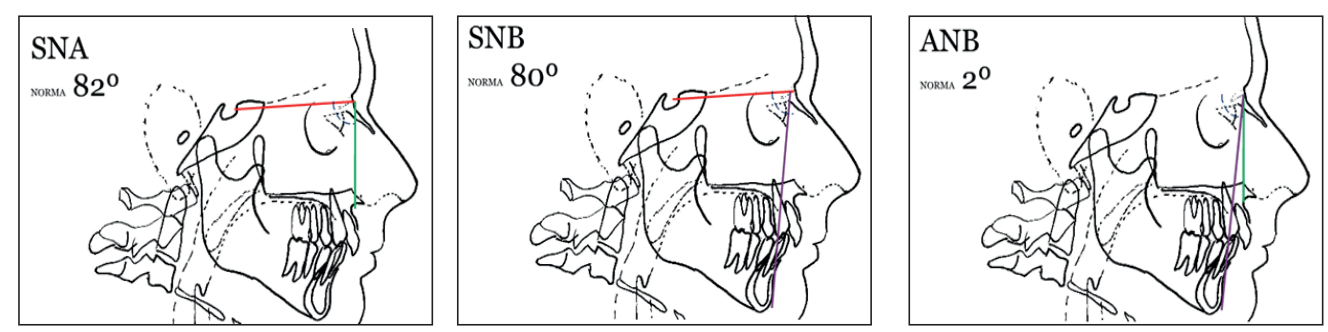

Fig. 6. Angles SNA, SNA et ANB et leurs valeurs normales. Fig. 6. SNA and SNB angles and their normal values.

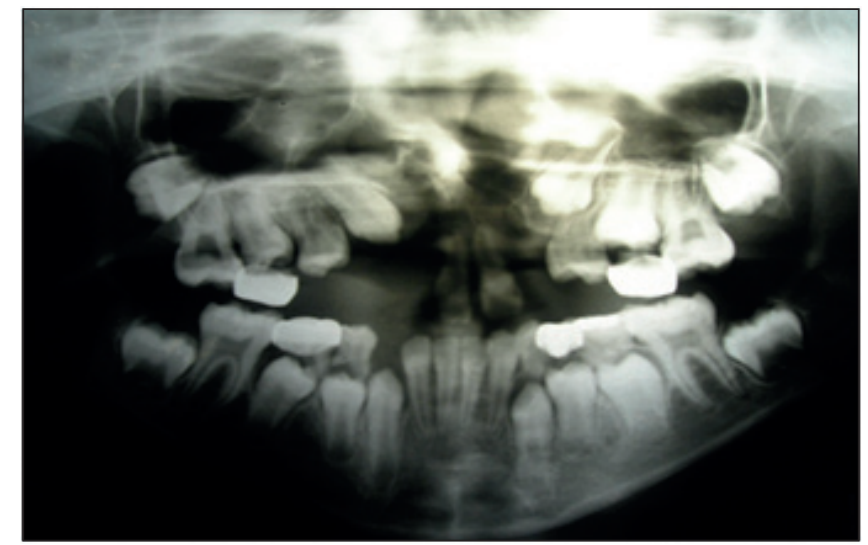

Fig. 7. Radiographie panoramique.

Fig. 7. Panoramic radiography.

photographies, les modèles, les radiographies etc. À ce stade, on évalue le profil, la compétence labiale, l'occlusion dentaire, la symétrie faciale, les sinus maxillaires, le squelette, la classe dentaire et la classe squelettique avec l'aide de la céphalométrie. Les modèles sont utilisés pour fabriquer non seulement l'appareil de notre choix, mais aussi pour effectuer un montage sur articulateur semi-adaptable pour visualiser l'objectif du traitement, en simulant l'intervention chirugicale sur les modèles et en confectionnant un guide chirugical.

L'objectif de cette étape est de corriger la malocclusion, puis de décompenser l'occlusion pour préparer et coordonner les arcades et la stabilité occlusale. On utilise un appareil orthodontique fixe ; la technique et la biomécanique utilisées varient avec le spécialiste en orthodontie (Fig. 8). Généralement,
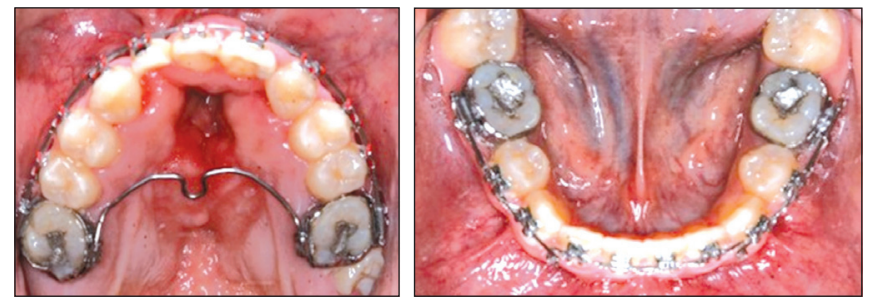

Fig. 8. Appareil orthodontique avec barre palatine. Fig. 8. Orthodontic appliance with transpalatal bar.

il faut compter 24 mois avant d'arriver à une relation adéquate entre les dents et les arcades en vue de l'intervention de chirurgie maxillo-faciale. Puis, on réévalue le cas et on réalise une étude céphalométrique pour décider le type d'intervention que l'on doit réaliser et pour savoir de combien de millimètres il faut avancer le maxillaire pour corriger la relation maxillomandibulaire (Fig. 9).

Avec le modèle des deux arcades, on contrôle l'occlusion pour éviter toute interférence occlusale et on réalise un montage sur articulateur pour obtenir la relation maxillomandi-bulaire par rapport à la base du crâne. On réalise l'intervention chirurgicale sur les modèles montés sur articulateur et on confectionne le guide occlusal chirurgical qui servira de référence per-opératoire.

\section{Orthodontie postchirurgicale}

Cette étape s'étend du $3^{\mathrm{e}}$ jour postopératoire jusqu'à l'obtention d'une occlusion fonctionnelle et esthétique, stable.

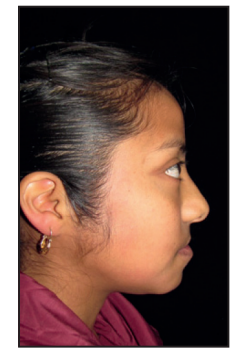

a

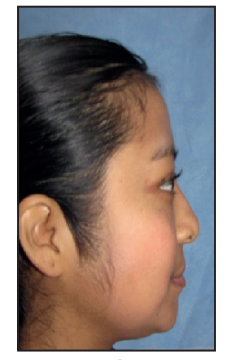

b

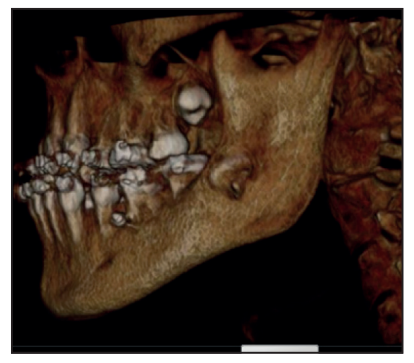

C

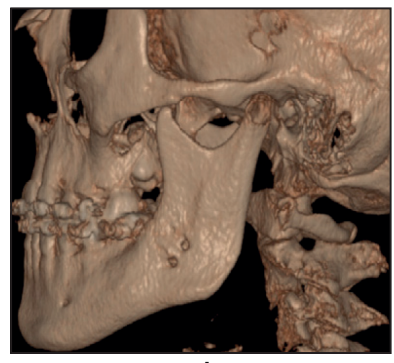

d

Fig. 9. Relation maxillo-mandibulaire avant $(A$ et $C)$ et après $(B$ et $D)$ l'intervention de chirurgie maxillo-faciale. Fig. 9. Intermaxillary relationship before $(A \& C)$ and after $(B \& D)$ maxillofacial surgery. 
Parfois, il est nécessaire d'utiliser des élastiques intermaxillaires pour maintenir l'occlusion, orienter les mouvements mandibulaires et contrecarrer les forces musculaires qui pourraient provoquer une récidive $(21,22)$. Il est important de ne pas utiliser les élastiques de manière prolongée pour éviter de créer une pseudo-arthrose. Ensuite, on réalise le dernier mouvement orthodontique pour terminer le traitement. Lorsque la stabilité des segments osseux est effective, on reprend les modèles d'étude et une radiographie panoramique pour analyser l'occlusion, et le parallélisme radiculaire avant de procéder à la dépose des appareils et la pose des arcs de contention. Si nécessaire, on réfère le patient à un confrère effectuer la réhabilitation prothétique.

\section{Conclusion}

Il a été démontré que l'application précoce de ces appareils et accessoires diminue le nombre d'interventions chirurgicales et améliore l'aspect physique du patient, ce qui a un retentissement psychologique et émotionnel favorable sur l'enfant et les parents.

Le traitement multidisciplinaire et dynamique donne la possibilité de soigner les patients présentant une FLP et d'éduquer les parents en leur donnant une information adéquate et orientée qui permet, avec la participation de toute l'équipe, d'améliorer la vie de ces patients.

\section{Conflits d'intérêt : aucun}

\section{Références}

1. Berkowitz S. Cleft lip and palate diagnosis and a management, 2nd ed. Springer, London, 1996.

2. Haas AJ. Palatal expansion: just the beginning of dentofacial orthopedics. Am J Orthod 1970;57:219-55.

3. Kernahan DA, Stark RB. A new classification for cleft lip and palate. Plast Recons Surg 1958;22:435.

4. Ortiz-Posadas MR, Vega-Alvarado L, Maya-Behar J. A new approach to classify cleft lip and palate. Cleft Pal Craniofac J 2001;38:545-50.

5. Berkowitz S, Duncan R. Timing of cleft palate closure should be based on the radio of the area of the cleft to that of the palatal segments and not on age alone. Plast Recons Surg 2005;115:1483-99.

6. Evans CA. Orthodontic treatment for patients with clefts. Clin Plast Surg 2004;31:271-90.
7. Grayson BH, Santiago PE, Brecht LE, Cutting CB. Presurgical nasoalveolar molding in infants with cleft lip and palate. Cleft Palate Craniofac J 1999;36:486-98.

8. Grayson BH, Cutting CB. Presurgical nasoalveolar orthopedic molding in primary correction of the nose, lip and alveolus of infants born with unilateral and bilateral clefts. Cleft Palate Craniofac J 2001;38:193-8.

9. Yeow VK, Chen PK, Chen YR, Noordhoff SM. The use of nasal splints in the primary management of unilateral cleft nasal deformity. Plast Reconstr Surg 1999;103:1347-54.

10. Cutting C, Grayson B, Brecht L, Santiago P, Wood R, Kwon S. Presurgical columellar elongation and primary retrograde nasal reconstruction in one-stage bilateral cleft lip and nose repair. Plast Reconstr Surg 1998;101:630-6.

11. Kozelj V. Experience with presurgical nasal molding in infants with cleft lip and nose deformity. Plast Reconstr Surg 2007; 120:738-45.

12. Frankel R. Maxillary retrusion in Class III and treatment with the functional corrector III. Trans Eur Orthod Soc 1970;249-59.

13. Mc Namara JA Jr, Huge SA. The functional regulator (FR-3) of Fränkel. Am J Orthod 1985;88:409-24.

14. Ricketts RM, Bench RW, Hilgers JJ, Schulhof R. An overview of computerized cephalometrics. Am J Orthod 1972;61:1-28.

15. Steiner CC. Cephalometrics for you and me. Am J Orthod 1953;39:729-55.

16. Steiner CC. The use of cephalometrics as an aid to planning and assessing orthodontic treatment. Am J Orthod 1960;46:721-35.

17. Tweed $\mathrm{CH}$. The Frankfort-mandibular incisor angle (FMIA) in orthodontic diagnosis, treatment planning, and prognosis. Angle Orthod 1964;24:121-69.

18. Friede $\mathrm{H}$, Johanson B. A follow-up study of cleft children treated with primary bone grafting. 1 . Orthodontic aspects. Scand J Plast Reconstr Surg 1974;8:88-103.

19. Lilja J, Moller M, Friede H, Lauritzen C, Petterson LE, Johanson $B$. Bone grafting at the stage of mixed dentition in cleft lip and palate patients. Scand J Plast Surg Hand Surg 1987;21:73-9.

20. Johanson B, Ohlsson A, Friede H, Ahlgren J. A follow-up study of cleft lip and palate patients treated by orthodontics, secondary bone grafting and prosthetic rehabilitation. Scand J Plast Reconstr Surg 1974;8:121-35.

21. Delaire J. Confection du masque orthopédique. Rev Stomatol Chir Maxillofac 1971;72:579-84.

22. Delaire J. L'articulation fronto-maxillaire: bases théoriques et principes généraux d'application de forces extraorales postéroantérieures sur masque orthopédique. Rev Stomatol Chir Maxillofac 1976;77:921-30. 